\title{
Automates et créatures artificielles d'Héphaïstos : entre science et fiction
}

Alexandre Marcinkowski et Jérôme Wilgaux

\section{(2) OpenEdition \\ Journals}

Édition électronique

URL : https://journals.openedition.org/tc/1164

DOI : $10.4000 /$ tc. 1164

ISSN : 1952-420X

Éditeur

Éditions de l'EHESS

Édition imprimée

Date de publication : 1 décembre 2004

ISSN : 0248-6016

\section{Référence électronique}

Alexandre Marcinkowski et Jérôme Wilgaux, «Automates et créatures artificielles d'Héphaïstos : entre science et fiction», Techniques \& Culture [En ligne], 43-44 | 2004, mis en ligne le 15 avril 2007, consulté le 29 septembre 2022. URL : http://journals.openedition.org/tc/1164; DOI : https://doi.org/10.4000/ tc. 1164

Ce document a été généré automatiquement le 29 septembre 2022.

Tous droits réservés 


\title{
Automates et créatures artificielles d'Héphaïstos : entre science et fiction
}

\author{
Alexandre Marcinkowski et Jérôme Wilgaux
}

1 Par définition, un automate est une machine capable de se mouvoir et d'accomplir des actions d'elle-même, de son propre mouvement. Mais, dans l'imaginaire occidental contemporain, le terme ne se réduit pas à cette seule aptitude : s'y associe souvent une impression d'illusion et de tromperie. Activé par un mécanisme intérieur qui reste secret et mystérieux, l'appareil « imite les mouvements d'un être vivant $»^{1}$, « [il] cache la cause première de son mouvement et fait croire à son organicité " (Beaune $1980: 7$ ). Dès lors, dans notre époque marquée par le développement de l'automatisation et de la robotique ${ }^{2}$, les automates peuvent être célébrés en tant que symboles du progrès technique et pour leurs promesses émancipatrices, mais sont tout autant considérés comme une source d'inquiétude, interrogeant l'homme sur sa propre humanité et sur la possible déshumanisation d'un monde futur dominé par les machines.

Du fait de leur ambiguïté, ils deviennent un objet d'études particulièrement approprié pour une réflexion sur les rapports entre techniques et vivant, entre nature et artifice (Lecourt 2003).

Une telle réflexion, qui pourrait sembler réservée aux spécialistes de science-fiction et aux observateurs du monde moderne, n'est pas sans intérêt pour les antiquisants. Comme le rappelait récemment Dominique Lecourt (2000), la fable de l'apprentisorcier ${ }^{3}$, popularisée au XXe siècle par un dessin animé de Walt Disney, est attestée pour la première fois dans la littérature européenne au IIe siècle de notre ère chez Lucien de Samosate ${ }^{4}$, et des automates sont déjà présents chez Homère, en association étroite avec Héphaïstos.

4 Dans le monde grec en effet, le divin forgeron joue un rôle fondamental dans la fabrication des créatures artificielles. Non seulement la tradition se place sous son patronage pour la pyrotechnie, les arts des métaux, mais les textes le perçoivent comme le démiurge par excellence, le dieu capable de créer des œuvres d'art tout à fait 
singulières. En cela, il est rejoint par Dédale, d'ailleurs présenté parfois par les auteurs anciens comme son descendant. Ces créations n'appartiennent pas cependant aux seuls mythes. Nos sources évoquent, à de nombreuses reprises, notamment aux époques hellénistiques et romaines, la présence de statues animées, de robots, de mécanismes automatiques lors de fêtes et processions religieuses ou, de manière plus générale, servant au culte des dieux.

5 Les Modernes ont peu écrit sur les créatures mécaniques dans l'Antiquité. Tellement peu qu'il faut se reporter à des ouvrages généraux sur l'intelligence artificielle pour en retracer l'histoire ${ }^{5}$. Ces ouvrages restent néanmoins trop descriptifs, souvent évasifs et lacunaires. Il nous paraît donc judicieux de rouvrir un dossier peu exploité par les hellénistes afin de nous interroger sur les raisons et les modalités de la fabrication de ces automates et autres créatures artificielles. Il s'agit ainsi de questionner les relations entre technique et magie dans la culture grecque, et de reprendre l'analyse des rapports entre divinités et êtres vivants, en nous attachant précisément aux œuvres merveilleuses d'Héphaïstos.

Héphaïstos, « l'illustre artisan »

6 Les dieux grecs se distinguent généralement par leur excellence et la plénitude de leur beauté; il n'en est pas de même d'Héphaïstos ${ }^{6}$, qui présente l'étrange particularité d'être un dieu difforme, infirme, un dieu boiteux ${ }^{7}$. Les qualificatifs qui le définissent sont sur ce point éloquents, même si leur interprétation a pu susciter quelques controverses: Héphaïstos est un dieu "aux pieds déformés, tordus, estropiés» (kullopodiôn, Iliade 18, 371; 20, 270; 21, 331), « boiteux des deux pieds ", " aux deux pieds retournés en dehors", "doué d'une direction double et divergente" (amphiguêeis ${ }^{8}$, Iliade 1, 607; 14, 239; 18, 383...; Hésiode, Théogonie 571; 579; 945; Les Travaux et les Jours 70; Bouclier 219; Frag. 209, 3).

7 L'explication de son infirmité varie suivant les sources. Selon la principale tradition, Héphaïstos boite depuis qu'il a été précipité de l'olympe par Zeus, ainsi châtié pour avoir pris la défense de sa mère, Héra, lors d'une dispute du couple divin. Après une chute d'un jour entier, il aurait été recueilli à Lemnos par les Sintiens (Iliade 1, 571sq.). Mais d'autres sources font de sa difformité non pas la conséquence mais la cause de cette chute : à sa naissance, sa mère, honteuse d'avoir enfanté un tel fils, l'aurait jeté du haut de l'olympe'.

8 Quoi qu'il en soit, Héphaïstos est ainsi présenté comme un dieu grotesque, objet de « rires inextinguibles»-par exemple lorsqu'il sert les dieux lors du banquet commun (Iliade 1,599-600)-, soumis aux railleries de ses congénères suite à une déambulation mal maitrisée comme à une insuffisance respiratoire, un dieu dont la vie est bien à plaindre (Iliade 18, 394 sq.; Odyssée 8, 266 sq.) mais qui peut également susciter l'admiration, car le divin forgeron, maître du feu, est un exceptionnel artisan ${ }^{10}$.

9 L'lliade, par exemple, nous informe qu'il a fabriqué pour les dieux leurs demeures olympiennes aux portes infranchissables (1, 606-607; 14, 166 sq.; 14, 338-339), plus particulièrement pour Zeus des portiques $(20,12)$, l'égide $(15,310)$, un sceptre par la suite transmis aux hommes (2, 101; voir également Pausanias 9, 40,11), un trône proposé par Héra au Sommeil $(14,238-240)$, une cuirasse pour Diomède $(8,195)$ et bien sûr un armement complet pour Achille (18, 369 sq.), comprenant le célèbre bouclier dont la surface était couverte de daidala (« œuvres d'art, merveilles artistiques » ${ }^{11}$ ), ce 
qui la rendait mouvante. À cela viennent s'ajouter des bijoux, colliers et autres objets, qu'il fabriquait dans la grotte marine de Thétis et d'Eurynomé $(18,400-405)^{12}$.

Si Héphaïstos est un piètre échanson, il est cependant capable de construire des objets qui se déplaceront d'eux-mêmes, des trépieds par exemple, capables de se rendre de leur propre mouvement (automatoi) à l'assemblée des dieux et d'être ainsi à leur disposition d'une merveilleuse manière ${ }^{13}$. Le dieu est en effet doué d'une aptitude singulière à imiter la vie et animer ses œuvres, aptitude qu'il révèle plus particulièrement en créant des automates. Outre les trépieds déjà signalés, les sources antiques lui attribuent la fabrication de servantes d'or qui l'assistent dans ses travaux (Iliade 18, 418-419), de six « charmeuses » faites d'or ${ }^{14}$, de deux chiens gardiens du palais d'Alkinoos ${ }^{15}$ ainsi que d'un autre destiné tout d'abord à Zeus ${ }^{16}$, de taureaux donnés à Aiètès ${ }^{17}$, de chevaux forgés pour le char des Cabires ${ }^{18}$, d'un aigle fabriqué pour Zeus ${ }^{19}$, et enfin d'un géant de bronze, Talos, laissé à Minos ou àEurope pour garder l'île de Crète ${ }^{20}$. À ces automates fabriqués sur le modèle d'objets usuels, d'animaux ou d'êtres humains, il faut ajouter les portes de l'olympe qui, dans l'Iliade, s'ouvrent d'elles-mêmes (automatai pulai) $^{21}$, ainsi que les soufflets de la forge, qui sous le commandement d'Héphaïstos semblent travailler de manière autonome (Iliade 18, 468-473).

Un monde sans ponos

11 La fonction de ces créations animées est évidente : auxiliaires des dieux, qui les ont parfois transmises aux hommes, elles ont été créées pour effectuer une tâche précise (rôle de gardien par exemple pour les chiens et Talos) qu'elles peuvent accomplir avec une perfection qui les rapproche des puissances divines. Elles partagent d'ailleurs avec ces dernières, par les métaux qui les composent (or, argent ou bronze), une immortalité de fait ${ }^{22}$ ou du moins une résistance particulièrement redoutable.

Dans une société divine où les Olympiens ne sont supposés connaître ni peine ni contrainte, ces créatures artificielles remplacent en quelque sorte les esclaves des sociétés humaines en se chargeant des travaux les plus répétitifs et les plus pénibles, des tâches les plus rébarbatives: les portes s'ouvrent d'elles-mêmes, les trépieds se rendent de leur propre mouvement au banquet des Olympiens, des servantes assistent Héphaïstos. Elles rendent ainsi possible cette existence idyllique que décrit Hésiode dans son évocation de la race d'or, du « temps de Kronos ${ }^{23}$, période durant laquelle la première race humaine vivait dans la communauté des dieux et menait, à leur exemple, « une vie préservée de souffrances, loin à l'écart des malheurs et des peines » ${ }^{24}$.

13 En ce temps-là, les travaux agricoles ne demandaient pas de grands efforts, car les productions se développaient spontanément, sans travail : c'est "d'elle-même » (automatê) que « la terre qui donne la vie [...] tendait ses fruits abondants » (v. 117-118; cf. Platon, Politique, 271d-272b) ${ }^{25}$.

14 De ce point de vue, la description des servantes d'Héphaïstos est particulièrement significative : jeunes, en or, elles incarnent parfaitement la richesse, la beauté, la force, la vitalité de cet âge d'or révolu pour les humains ${ }^{26}$. Et lorsque Aristote, dans un passage célèbre de la Politique, mentionne les créations d'Héphaïstos ${ }^{27}$, il fait tout autant référence à ce passé qu'il imagine un monde où l'esclavage serait inutile :

«Si chaque instrument était capable, sur une simple injonction, ou même pressentant ce qu'on va lui demander, d'accomplir le travail qui lui est propre, comme on le raconte des statues de Dédale ou des trépieds d'Héphaïstos, lesquels dit le poète : "Se rendaient d'eux-mêmes à l'assemblée des dieux", si, de la même manière, les navettes tissaient d'elles-mêmes, et les plectres pinçaient tout seuls la 
cithare, alors, ni les chefs d'artisans n'auraient besoin d'ouvriers, ni les maitres d'esclaves. » (1, 4, 1253b33-1254a1, trad. J. Tricot, Vrin) esclaves ${ }^{29}$ ou d'une manière plus générale les êtres vivants; elles effectuent leur tâche, nous l'avons dit, à la perfection et surpassent assurément les êtres mortels. Nul ne peut échapper par exemple au chien forgé par Héphaïstos et qui est à l'origine de la race des molosses $^{30}$. Quant à Talos, il est capable de faire le tour de la Crète trois fois par jour ${ }^{31}$ et de s'opposer à toute intrusion sur l'île; il faudra toute l'ingéniosité et la magie de Médée pour en venir à bout ${ }^{32}$. Particulièrement efficaces, ces automates restent cependant limités dans leurs compétences. Car, si les trépieds peuvent remplacer Héphaïstos pour servir les dieux lors des banquets, si Hephaïstos peut s'appuyer sur des robots à la matière inaltérable et aux gestes ultra-rapides ${ }^{33}$ ou être assisté de ses soufflets, nul mieux que lui n'est à même de réaliser les ouvrages demandés; il n'existe pas d'automate pouvant le remplacer dans cette tâche et le divin forgeron est par ailleurs la seule divinité capable de fabriquer de telles merveilles. Afin d'approfondir nos analyses, entrons dans le détail des textes.

Le corps du dieu en ses pensées

17 Lorsque Thétis vient trouver l'hospitalier boiteux dans son logis d'airain, la déesse assiste à une incroyable scène. Héphaïstos, en sueur, s'affaire à la forge pour fabriquer simultanément vingt trépieds en or qu'il a munis de roulettes afin qu'ils puissent faire l'aller-retour entre le palais des dieux et son atelier. Ne manquent que les anses ouvragées (ouata daidalea) attendant d'être rivetées par le dieu (Iliade 18, 373-379) ${ }^{34}$. Travail exigeant que seul peut accomplir un dieu aux «savantes réflexions » (iduiêisi prapidessi, Iliade 18,380 ).

18 L'expression iduiêisi prapidessi est éclairante. Exclusivement réservée à Héphaïstos chez Homère (Delcourt 1982 : 53-54), on la retrouve par exemple dans l'Iliade 18, 481-482, en rapport avec le bouclier d'Achille composé de cinq plaques (deux de bronze, deux d'étain, une d'or = Iliade 20,271), ou encore en Odyssée 7, 91-94 à propos des deux chiens d'or et d'argent, gardiens du palais d'Alkinoos ${ }^{35}$. L'adjectif iduîa signifie «savant, expert », et prapis a été souvent interprété comme désignant le diaphragme puis le siège de l'intelligence. L'analyse approfondie que Richard B. Onians propose de ce terme montre qu'au pluriel, assimilé aux phrenes ${ }^{36}$, il peut désigner les poumons et, en tant que tel, le siège du thumos, du "souffle", de la conscience, des pensées et des émotions (Bremmer 1983 : 62). Dans un article récent, Françoise Frontisi-Ducroux est revenue sur l'expression iduiêisi prapidessi. Ayant analysé ses occurrences en dehors du champ lexical homérique, elle remarque leur association avec le système visuel et propose de traduire les deux mots par «diaphragme clairvoyant, visionnaire» 
(Frontisi-Ducroux 2002 : 478-479), rapprochant ainsi le forgeron de l'aède en soulignant la relation étroite entre la respiration du dieu et l'acte créatif.

«Cet organe est en l'occurrence le système respiratoire, plus précisément sans doute la partie qui l'active et le régule, une zone qui chez les dieux est douée de la faculté de voir ce que les yeux ne voient pas, un diaphragme "clairvoyant", ou "visionnaire". Ce que confirme le développement exceptionnel de son torse et aussi l'accent mis sur les soufflets, seconds poumons externes et auxiliaires dociles du dieu... et sur son propre essoufflement. Le thorax d'Héphaïstos, suant et soufflant, est une vaste boîte à images, le siège de son inspiration créatrice, l'organe de ce qui sera, bien plus tard, l'imagination. [...] Le rôle attribué aux prapides dans la création artistique, telle qu'elle est mise en scène au chant 18 de l'Iliade, à travers la fabrication par Héphaïstos d'un ouvrage figuratif, nous semble relever d'une représentation proprement aédique. » (Frontisi-Ducroux 2002 : 479-480)

Quoi qu'il en soit, nous garderons à l'esprit l'image du couple diaphragme/poumons qui se soulève et s'affaisse au rythme de la respiration, à l'image des soufflets de la forge héphaïstienne qui croissent et décroissent. Les «savantes réflexions» du démiurge s'inscrivent dans une manifestation sensorielle de la membrane frontière qu'est le diaphragme et, de manière plus générale, les descriptions homériques établissent une continuité, une symbiose, entre l'attitude du dieu, ses instruments et ses créations. Le forgeron, suant, soufflant, s'empresse comme le font ses servantes, tourne sur luimême et roule (helissomenon, 18, 372), comme si ses jambes grêles aux pieds tordus étaient comparables aux roues dont il a muni les trépieds. Avec ses membres inférieurs atrophiés et son buste puissant, ne présente-t-il pas d'ailleurs une certaine similitude avec ces mêmes trépieds? Pour les scholiastes, ce dieu qui tourne sur lui-même, à la mètis courbe, sinueuse ${ }^{37}$, est à l'image du feu qui tourbillonne ${ }^{38}$, et la constance de cette analogie fait de l'univers de la forge un tout unique, présentant les mêmes propriétés, dont le feu et le souffle fournissent les clés ${ }^{39}$.

Nature des automates

Apparaissant ainsi comme des prolongements corporels du dieu, ces créations ont cependant une réelle autonomie qui n'est pas sans susciter une certaine perplexité. Les remarques d'Eustathe, Commentaire de l'liade 18, 473-477 (trad. J. Wilgaux), sont de ce point de vue particulièrement significatives ${ }^{40}$ :

« Il était en effet merveilleux que les trépieds se déplacent d'eux-mêmes, comme s'il s'agissait d'objets disposant de leur propre mouvement et avançant grâce à des roues installées à leur base, des poulies, qui, comme il convient, auraient été insérées dans les pieds des chaudrons, de sorte qu'ils puissent entrer là où se tenait le rassemblement des dieux, l'assemblée des Olympiens, puis retourner à leur point de départ, chez eux, à la manière d'êtres animés (empsukhôn). Tels sont supposés être les objets fabriqués par Héphaïstos. Il faut savoir en effet que le poète dépeint ces trépieds animés d'une manière merveilleuse, se mouvant par eux-mêmes, comme s'ils se déplaçaient grâce aux petites roues sur lesquelles ils reposent. C'est pourquoi l'un des Anciens -il s'agit de Denys- a supposé que les êtres forgés par Héphaïstos sur le bouclier d'Achille, qui seront évoqués par la suite, se meuvent également d'eux-mêmes, même s'il a été contredit, dit-on, par Aristonikos. Il faut remarquer que les Anciens établissent de telles conjectures et tiennent de tels propos parce qu'à cause de la très grande habileté de ceux qui travaillent le métal, leurs représentations semblaient presque animées. C'est ce que l'on raconte à propos des Rhodiens, dont l'île autrefois bénéficiait d'une telle technique, et où les figurines avaient à ce point l'apparence du mouvement qu'on les attachait par des chaînes pour qu'elles ne puissent bouger et s'enfuir. $»^{41}$ 
21 d'un embarras : comment penser l'animation? Les analogies proposées par Homère ou Hésiode sont en effet troublantes. Citons par exemple le vers 418 du chant 18 de l'iliade, dans lequel il est dit que les servantes d'Héphaïstos sont « semblables à de jeunes êtres vivants " (zôêisi neênisin eioikuiai). En cela, elles peuvent être comparées aux femmes qui, représentées sur le magnifique bouclier forgé pour Héraklès, « du haut des murailles solides, forgées d'airain, hurlaient et se lacéraient le visage, comme vivantes (zôeisin ikelai) - par l'art d'Héphaïstos, l'artisan illustre » (Hésiode, Le Bouclier 242-244, trad. Ph. Brunet, LGF), ou bien encore aux combattants représentés sur le bouclier destiné à Achille, qui se battent « comme des mortels vivants » (Iliade 18, 539). Pour la plupart des commentateurs, ces créations ne sont pourtant pas à mettre sur le même plan : l'animation des scènes représentées sur les boucliers forgés par Héphaïstos, que ce soit dans l'Iliade ou chez Hésiode, relève de la convention artistique et souligne l'extraordinaire qualité du travail du dieu ${ }^{42}$, tandis que les créations précédemment inventoriées (jeunes servantes, chiens, trépieds...) sont réellement animées, peuvent se déplacer et effectuer de véritables tâches. Elles sont même à ce point comparables à des êtres vivants qu'elles sont capables de se reproduire, comme c'est le cas d'un chien forgé par Héphaïstos, qui serait à l'origine de races canines actuelles :

«On dit que les chiens de Khaonie et les molosses sont les descendants d'un chien qu'Héphaïstos forgea à partir du bronze de l'île de Dèmonèsos et auquel il insuffla une âme; il le donna en présent à Zeus... $»^{43}$

Dès lors, quelle est la nature exacte de ces automates ? En quoi consistent-ils ? Comment sont-ils animés ? Dans tous les cas, Héphaïstos donne à l'objet forgé la capacité réelle de se mouvoir et d'agir de lui-même. Mais quel en est le processus précis? À lire Pollux $(5,38)$, par exemple, l'opération semblerait reposer sur l'adjonction d'une âme (psukhè) à un métal forgé en respectant une forme conventionnelle (en l'occurrence celle d'un chien), et c'est le même vocabulaire que nous retrouvons dans de nombreux passages évoquant les créations d'Héphaïstos, comme en témoigne la citation précédente d'Eustathe ${ }^{44}$. Le terme français retrouve donc ici son étymologie, " animer » aurait bien le sens de " doter d'une âme » (psukhè, empsukhos).

Mais ces textes sont pour l'essentiel tardifs, et ni le terme psukhè, ni notre opposition du corps et de l'âme, ne se retrouvent chez Homère en relation avec les automates ${ }^{45}$. De manière plus générale, à l'époque archaïque, un être animé est conçu comme un tout dans lequel il est en réalité impossible de distinguer le spirituel du corporel. Le cas de Talos, par exemple, est significatif ${ }^{46}$. Il s'agit donc d'un homme de bronze qui, selon les traditions, appartiendrait à la race de bronze (Vernant 1985 : 32) ou bien aurait été forgé par Héphaïstos. Ce matériau le rend indestructible, mais ce gardien de l'île de Crète possède néanmoins un point faible, sa cheville :

«Son corps et ses membres étaient faits d'un airain infrangible; mais à sa cheville, sous le tendon, il avait une veine pleine de sang (haimatoessa) et c'est de la fine membrane qui la fermait que dépendaient sa vie et sa mort.» (Apollonios de Rhodes, Argonautiques 4, 1645-1648, trad. E. Delage et Fr. Vian, CUF)

Selon Apollodore ${ }^{47}$, il possède une seule veine, allant du cou jusqu'à la cheville, dans laquelle coule l'ikhôr, et qui est fermée par un clou : parvenir à enlever ce clou, c'est permettre au liquide de s'écouler et donc ôter la vie. 

apparaître une vilaine coupure d'où s'échappait du "sang " (ikhôr) qui avait la couleur du plomb fondu. Le géant de bronze s'effondra tel une statue, brisé à la base, dans un terrible fracas (Argonautiques 4, 1679-1688). Ce sang qui n'est pas du sang, comme l'avait bien vu par ailleurs Nicole Loraux (2003 : 486-489), marque la frontière entre le statut des mortels et des immortels. Dépassant l'aspect métaphorique du sang, Arthur B. Cook (1914, t. 1, 723-724), puis F. Frontisi-Ducroux $(2000: 127)^{48}$ ont vu dans la constitution de Talos une référence à la technique de la fonte "à cire perdue ", le métal en fusion remplaçant dans le moule la cire qui elle-même, au fur et à mesure qu'elle fond, s'écoule par des orifices. En ce sens, la technique de production, seule à même de produire des œuvres métalliques d'une seule pièce, rend indissociables la robustesse externe de l'ensemble, son

invulnérabilité apparente, et sa fragilité interne, son vide, ainsi que la présence d'une "veine", nécessaire à sa création mais désormais menacée d'un écoulement inconsidéré.

Quoi qu'il en soit, nous nous trouvons toujours en présence de l'association d'une forme métallique et d'un principe vital, ici de l'ikhôr ou du sang, un principe lui-même corporel donc, et qui n'a pas été ajouté mais au contraire est à l'origine de la création de l'être animé. Les automates d'Héphaïstos sont en vérité vivants : un ou des principes vitaux sont présents en eux et, comme nous l'avons vu, ils possèdent la capacité de se perpétuer.

Semblances de femmes

Les détails, particulièrement riches d'enseignement, donnés par l'Iliade à propos des servantes du divin forgeron, vont dans le même sens et nous permettent de compléter nos remarques précédentes :

"[...] des servantes s'empressent sous leur maitre,

elles sont en or, semblables à de jeunes êtres vivants;

dans leurs phrenes se trouve une pensée réfléchie (noos), ainsi qu'une voix (audê)

et qu'une force (sthenos), et des dieux immortels elles ont appris des travaux.

Elles s'activent sous leur maître [...]. » (Iliade 18, 417-421, trad. littérale, J. Wilgaux)

Nous avons déjà évoqué l'expression employée au vers 418, «semblables à des êtres vivants». Pour mieux la comprendre, il convient de reprendre ici les analyses développées par Jean-Pierre Vernant (1996a = Vernant 1996b), ainsi que par Daniel Saintillan (1996 : $343 \mathrm{sq}$.) et Pierre Judet de La Combe (1996), en rappelant tout d'abord que ces vers ont un parallèle célèbre; en effet, dans Les Travaux et les jours (v. 60-64, trad. Ph. Brunet, LGF), la description de la création de Pandore présente de nombreuses similitudes $^{49}$ :

« [Zeus] invita le célèbre Héphaïstos à pétrir au plus vite

de la glaise et de l'eau, à y mettre une voix humaine (audê)

et la force (sthenos) à y joindre un visage divin de déesse,

une charmante beauté virginale. Athéna, par la suite,

lui apprendrait les travaux du tissage aux couleurs innombrables.»

Vers auxquels fait écho le passage suivant de la Théogonie (v. 571-572; trad. Ph. Brunet, LGF) :

«Le très illustre artisan, le Boiteux, façonna dans la terre,

et Zeus le voulait -un être semblable à la vierge pudique. »

Mais revenons au texte de l'Iliade. Parmi les termes employés, l'un des plus importants pour comprendre les conceptions homériques reste le noos. On rencontre en effet ce 
terme tout au long des poèmes homériques ${ }^{50}$. R. B. Onians en rattache l'étymologie au verbe neomai, « je vais ", indiquant ainsi un mouvement particulier ${ }^{51}$, et définit le noos comme un dessein dynamique, une projection de la conscience, une action intellectuelle, qui ne serait attribuable à aucun organe physique ou palpable (Onians 1999 : 107). Bruno Snell, dans sa tentative de définition de la conception de l'homme homérique, parle du noos comme étant «quasiment l'œil intérieur qui fait preuve de lucidité », et l'interprète comme la faculté d'avoir une pensée réflexive, claire (Snell 1994 : 31).

31 L'homme homérique ne bénéficie pas d'une conception unifiée de l'âme. Comme B. Snell (1994: 25) l'a montré, les représentations de l'âme chez Homère restent inséparables de la représentation du corps et inversement. En schématisant quelque peu, le moi homérique se compose de la psukhè, cette âme-souffle qui quitte le corps au moment de la mort, et du couple thymos-noos, ou âme-sang, qui a la zone du diaphragme et du cœur pour centre des fonctions intellectuelles et sensitives. En indiquant que les jeunes servantes sont dotées d'un noos, Homère précise ainsi ce qui permet de les assimiler à des êtres vivants et plus encore, bien évidemment, aux humains.

En fait, les ressemblances entre déesses, mortelles et créations d'Héphaïstos évoquées dans le corpus homérique et hésiodique s'agencent comme dans un jeu de miroirs pour déboucher sur l'autoréférentialité : Hélène ressemble aux déesses immortelles (Iliade 3, 158; cf. Trav. 62) et Nausicaa à Artémis (Odyssée 6, 151-152), tandis que Pandore ressemble tout à la fois aux déesses et à une « vierge pudique » (parthenos aidoiê), dont elle est le prototype ${ }^{52}$.

Des réflexions menées par les philologues et les historiens sur ces copies de copies qui ne sont en réalité que des copies d'elles-mêmes, ressort l'idée fondamentale qu'il serait vain d'opposer chez Homère et Hésiode l'apparence à l'essence, ou d'y rechercher une dénonciation de l'image comme artifice, comme faux-semblant. Les jeunes servantes ne peuvent être considérées comme de simples simulacres car leur « semblance » renvoie à leur identité propre; citons ici J.-P. Vernant (1996a : 392-393) :

«Cette semblance première à travers laquelle se fait reconnaître pour chacun son identité n'est pas de l'ordre d'une imitation trait pour trait, mais d'une congruence par rapport à une norme, d'une évaluation par rapport à un modèle exemplaire. Similitude à soi donc, constitutive de l'identité et dont l'eidôlon présente comme un duplicata, au même titre que les enfants par rapport au père qui, pour les engendrer, imprime son modèle, son tupos, dans la matrice de l'épouse, afin qu'ils soient "semblables à lui", eoikota tekna (Trav. 182, 235), ou que Pandora façonnée dans la glaise par Héphaïstos à la semblance d'une parthenos (Trav. 71, Théog. 572), c'est-à-dire de ce qu'elle sera, dès lors que la similitude avec elle-même se trouvera en elle effective; et cette identité féminine qu'assume Pandora en s'assimilant au modèle de la parthenos renvoie à son tour à une autre semblance : par la beauté de son jeune corps de vierge, de ses vêtements, de ses parures, de sa couronne, par la kharis, la puissance de séduction qui en émane, la parthenos est elle-même "à l'image des déesses immortelles". » (Trav. 62) ${ }^{53}$

Dans le cas de Pandora comme dans celui des servantes d'or, Héphaïstos n'imite donc pas les mortelles mais les reproduit, et ces créations doivent bien être considérées comme vivantes, comme le prouve la présence en elles d'une intelligence (noos), d'une voix (audê), d'une force (sthenos) ${ }^{54}$, et d'une aptitude aux travaux féminins.

Mais cette "semblance " s'accompagne d'une série d'écarts qui rendent possible une certaine hiérarchisation des êtres : les jeunes filles d'or sont «semblables à des êtres vivants » et non à des déesses immortelles, inégalables en vérité malgré 
l'immarcescibilité des matériaux utilisés, ce qui souligne leur caractère subalterne, leur rôle de servantes, d'auxiliaires du dieu; mais en même temps, elles peuvent être considérées comme supérieures aux mortelles. Si comme Pandora elles ont en effet pour créateur Héphaïstos, elles sont en or, et non composées de glaise et d'eau, et par ailleurs ne recèlent en elles aucun piège, aucune tromperie. Parfaites dans leur fonction, leur apparition dans le monde des dieux n'est en aucun cas l'indication d'une déchéance, bien évidemment, mais au contraire est révélatrice de la puissance des dieux et de la capacité démiurgique d'Héphaïstos, voire de la supériorité de la création artisanale sur la nature, puisque ces automates sont supérieurs aux mortels et qu'en se perpétuant par génération (transmettant ainsi leur "semblance», leurs caractéristiques propres, leur force par exemple dans le cas des molosses) ils connaissent alors une dégénérescence, qui se traduit par la perte de leur composition métallique et de leur immortalité.

La fabrique du vivant

Cette capacité d'Héphaïstos à transformer la matière inerte en matière animée peut s'interpréter en termes de magie, d'enchantement, et c'est ainsi que l'entendent la plupart des historiens (Wathelet 2000), à l'instar de Marie Delcourt. Pour cette dernière, les caractéristiques archaïques d'Héphaïstos trouvent leur origine dans la figure mythique d'un dieu magicien, «tout autant capable de lier et d'immobiliser que d'animer l'immobile »; elle poursuit ainsi :

«Si l'on voit en Héphaistos un magicien qui a payé sa science de son intégrité corporelle, tous les épisodes de sa légende s'éclairent et forment un ensemble cohérent. » (Delcourt 1982 : 11)

Dans la continuité de ces analyses, Christopher Faraone a plus particulièrement attiré l'attention sur les similitudes, du point de vue de la fonction, de la géographie et du vocabulaire, présentées par certaines des créations héphaïstiennes, en particulier le chien d'or d'Alkinoos et Talos. L'homme de bronze et le chien d'or, tous deux destinés à un rôle de gardien, proviennent de Crète et sont parvenus à Minos soit en cadeau d'Héphaïstos lui-même, soit indirectement, après être passés de mains en mains. Par ailleurs, tant Pollux que Simonide de Céos (Faraone 1987:260-261), premier poète à avoir mentionné Talos, emploient le terme empsukhos ou psukhè pour signifier l'animation de ces deux créatures. C. Faraone établit un parallèle avec un fragment d'Alcée où il est question d'Héphaïstos fabriquant « un lion de bronze dans lequel il plaça des drogues secourables aux humains" (frag. 306Ea, col. I, trad. G. Liberman, CUF). Ce lion d'un genre particulier, Macar l'emporta à Lesbos pour le cacher, assurant ainsi le salut de l'île. Faraone signale que l'animation des créatures humaines ou animales est à usage apotropaïque et que la pratique faisant intervenir des pharmaka dans les statues était une spécialité des magiciens et théurges de l'époque impériale. Cependant, le fragment ne précise en rien que le lion de bronze pourrait être automatos ou empsukhos. Plus généralement, rien dans l'œuvre homérique ne laisse entendre qu'Héphaïstos est un sorcier, un goês ou un magos, ou du moins les mots ne sont pas employés; et les descriptions qui sont faites de son activité ne ressemblent en rien aux comportements attendus des magiciens. Si l'hypothèse des origines magiques des pouvoirs d'Héphaïstos permet de comprendre de nombreux épisodes mythologiques relatifs à ce dieu, il n'en reste pas moins, comme M. Delcourt le reconnaissait ellemême $(198215)^{55}$, que cette dimension est peu explicite dans les textes homériques, où Héphaïstos est célébré pour ses prouesses techniques, son habileté remarquable et son ingéniosité qui lui permettent de transgresser les limites habituelles de la création 
artisanale ${ }^{56}$. Les trépieds, par exemple, ne se mettent pas à marcher comme par magie, mais se déplacent grâce aux roues ajoutées par le dieu, c'est-à-dire grâce à la mise en place d'une mécanique. Il faut reconnaître que l'impuissance des Modernes à rendre compte de l'intrusion de la technique dans les mythes a facilité l'application de l'étiquette "magie » à tout événement extraordinaire. En fait, l'impression donnée dans l'Iliade est que l'infirmité du dieu n'est pas la contrepartie de son art, mais qu'elle rend nécessaire la création d'assistants autonomes, donc d'automates, aptes à l'aider dans son travail éprouvant et à lui permettre de se déplacer; elle est par ailleurs révélatrice des caractéristiques propres de la mètis du forgeron: "C'est la puissance d'Héphaïstos que souligne le privilège d'être doué d'une direction double et divergente. » (Detienne \& Vernant 1974 : 259-260).

Si Athéna et Héphaïstos jouent tous deux un rôle essentiel dans le domaine des techniques, le Boiteux est apparemment le seul à pouvoir animer ses créations ${ }^{57}$. Pour expliquer cette singularité, nous pouvons renvoyer ici à nos propos précédents relatifs à la courbure, à la sinuosité, associées à la démarche du dieu ainsi qu'aux automates, à sa relation étroite au feu et au souffle ${ }^{58}$, principes vitaux fondamentaux, qui expliquent déjà en partie sa puissance démiurgique. Il nous faut insister davantage, cependant, sur ses domaines d'intervention : en tant que forgeron, et à l'occasion potier, Héphaïstos ne se contente pas de travailler la matière, de l'agencer de telle ou telle manière, mais transforme les matériaux utilisés, procède à des mélanges, des fusions, des alliages. Son action est dès lors comparable à celle de la nature qui, dans le processus de génération humaine par exemple, parvient à créer de la chair et des os à partir de sperme et de sang. C'est pourquoi Héphaïstos est la seule divinité apte à fabriquer des automates, et que ceux-ci sont tout aussi vivants que les humains ou les animaux. Ils procèdent en vérité des mêmes principes, de la même transformation de substances corporelles par le souffle et le feu.

Les relations entre techniques et vivant dans les mythes grecs révèlent ainsi toute leur complexité. Héphaïstos ne crée pas d'êtres fabuleux; il s'inspire pour ses créations du monde tel qu'il est, et ses productions peuvent être considérées comme des reproductions perfectionnées. Mais ces automates, malgré leurs qualités, ne menacent pas le monde des hommes; ils ne sont pas en effet posthumains, comme le seraient les cyborgs et autres clones imaginés aujourd'hui, mais pré-humains, à l'instar de Pandore. S'ils se perpétuent, c'est en respectant désormais le processus de génération, et c'est donc la nature qui se charge de reproduire les êtres artificiels. Selon notre interprétation, lesautomates d'Héphaïstos sont vivants non pas tant parce qu'ils imitent la vie par magie, mais parce que technique et monde vivant obéissent aux mêmes principes tout en divergeant dans leurs réalisations.

Les récits mythologiques évoquant la création d'automates nous livrent donc l'image d'un monde unifié, dans lequel il serait vain d'opposer de manière générale le naturel au surnaturel ou bien encore à l'artificiel (Padel 1992). Tout processus de création, de reproduction, repose sur les mêmes principes, sur les mêmes réalités physiques, mais les résultats obtenus présentent des différences de degrés, en fonction des compétences variables de leur auteur. C'est ainsi que les créations d'Héphaïstos ne peuvent égaler la génération des Olympiens mais se révèlent supérieures aux êtres mortels. Pour tout artisan humain, le divin forgeron représente par conséquent un modèle indépassable, fixant les limites mais aussi l'ambition de toute œuvre portée au plus haut point de perfection, à savoir l'animation, le don de vie. La nature, par le processus de 
génération, se montre particulièrement apte à transmettre la "semblance » des êtres, mais ses modalités ne diffèrent guère de celles d'Héphaïstos, comme l'enseignèrent bien plus tard les Stoïciens, pour lesquels « la nature est un feu artiste (pur tekhnikon), qui avance méthodiquement en vue de la génération; c'est un souffle igné et artisan (pneuma puroeides kai tekhnoeides)» (Diogène Laërce, Vies et doctrines des philosophes illustres 7, 156, trad. R. Goulet, LGF) ${ }^{59}$.

Merveilleuses machines

41 Un mortel eut la réputation d'égaler Héphaïstos, dont il est considéré aujourd'hui comme un « doublet fonctionnel » (Frontisi-Ducroux 2000 : 94). C'est Dédale l'Athénien, qui, dans l'esprit des Grecs, fort de sa notoriété artistique et de son talent démiurgique, est le premier homme à avoir conçu des sculptures à l'apparence d'êtres vivants ${ }^{60}$. Dans une occurrence, nous le voyons également travailler en compagnie de deux Eros qui manient la scie et qui sont manifestement des automates ${ }^{61}$. Comme l'ont montré les travaux de Françoise Frontisi-Ducroux, reprenant en détail l'intégralité des sources, les œuvres de Dédale nous font cependant entrer dans le domaine du simulacre. Dans le monde des hommes, la volonté de rendre l'inerte animé dont témoignaient mythes et poésie se traduit par la réalisation physique d'automates en bois ou en métal, qui usent de mécanismes secrets pour donner l'illusion du vivant.

42 À suivre Pline l'Ancien, le plus vieil automate est l'œuvre de Canachos de Sicyone. Le sculpteur avait façonné un cerf qui, par le truchement d'un mécanisme, bondissait d'avant en arrière ${ }^{62}$. C'est à un contemporain de Platon, Archytas de Tarente, que l'on doit, selon une méthode mécanique, une colombe en bois qui volait ${ }^{63}$. Pausanias $(6,20$, 10-12) signale, à Olympie, un dauphin et un aigle aux ailes déployées en bronze qui, lorsque le responsable des courses hippiques manipulait un mécanisme placé dans l'autel, jaillissaient vers le sol pour le mammifère marin, vers le ciel pour le rapace. Lors des Grandes Dionysies de 308, Démétrios de Phalère produisit un escargot mécanique qui bavait (Polybe 12, 13, 11).

Ainsi l'invention de machines merveilleuses se développa pour devenir une branche spécifique de la mécanique hellénistique ${ }^{64}$. De nombreuses applications des principes pneumatiques, c'est-à-dire nécessitant la pression de l'air (pneuma), de la vapeur et de l'eau pour obtenir du mouvement, ont été réalisées par Ctésibios, Philon de Byzance ou Héron d'Alexandrie. Ctésibios avait mis au point des automates à musique en forme de rhyton, une pompe à incendie à valves et pistons. On trouve également décrits de nombreux automates dans les Pneumatika d'Héron, comme dans ses Automata, ouvrage aujourd'hui perdu où il précisait les mécanismes employés dans les machines de théâtre. Parmi d'autres inventions, mentionnons le distributeur automatique d'eau (Pneum. 1, 36), un temple en réduction dont les portes s'ouvrent d'elles-mêmes lors de l'activation du feu sacrificiel sur l'autel (Pneum. 1, 38) ou encore lors du son d'une trompette (Pneum. 1, 17).

Manifestations de la maîtrise technique de leur créateur, ces automates ne peuvent plus cependant être considérés comme des auxiliaires rendant possible un monde sans labeur; ne produisant rien par eux-mêmes, si ce n'est de l'émerveillement, ils n'ont pas été l'objet d'application « industrielle » et sont sans influence sur la vie économique.

D'Homère jusqu'à l'époque romaine, nos sources insistent sur l'importance de la reproduction du mouvement dans la figuration du vivant, et cette ambition débouche sur des réalisations concrètes qui relèvent du domaine de l'illusion : la nature peut être imitée, elle ne peut être égalée. Mais tel n'est pas le cas dans les récits mythiques, où 
ces merveilleuses machines sont de véritables êtres vivants. Le génie d'Héphaïstos rivalise en effet avec la nature puisque ses œuvres sont immortelles, inaltérables, parfaites de beauté et d'efficacité. Plus encore, certaines d'entre elles sont capables, à l'instar des êtres vivants, de se perpétuer et d'engendrer une descendance. Ce n'est que lorsqu'elles quittent l'Olympe et pénètrent le monde des hommes, que ces créatures artificielles s'exposent à la dégénérescence, à la mort, interrogeant la nature du vivant et la question de l'âme.

En créant des automates, Héphaïstos n'imite pas le vivant: il le fabrique, car le mouvement, la vie, procèdent de processus physiques, de mécanismes, qui relèvent de ses compétences, de sa mètis. En sublimant la technique par la figure mythique du divin forgeron, les sources grecques archaïques nous donnent ainsi à voir une nature ellemême technicisée.

Nous remercions chaleureusement Jean Yvonneau, Alain Bresson et Jean-Luc Jamard pour leurs précieux commentaires sur une version initiale de cet article.

\section{BIBLIOGRAPHIE}

Amartin-Serin, Annie

1996. La Création défiée. L’homme fabriqué dans la littérature. Paris : Presses Universitaires de France (« Littératures européennes »).

Argoud, Gilbert \& Jean-Yves Guillaumin

1997. Les Pneumatiques d'Héron d'Alexandrie. St Etienne : Presses universitaires.

Beaune, Jean-Claude

1980. L'Automate et ses mobiles. Paris : Flammarion (« Sciences humaines »).

Blaise, Fabienne, Julien Judet de la Combe \& Philippe Rousseau

1996. Le Métier du mythe. Lectures d'Hésiode. Lille : Presses Universitaires du Septentrion (Cahiers de Philologie, vol. 16, série Apparat critique).

Bolens, Guillemette

2000. La Logique du corps articulaire. Les articulations du corps humain dans la littérature occidentale. Rennes : Presses universitaires (« Histoire »).

Bremmer, Jan

1983. The Early Greek Concept of the Soul. Princeton : Princeton University Press.

Bresson, Alain

1979. Mythe et contradiction. Analyse de la VIIe Olympique de Pindare. Besançon : Annales littéraires de l'Université de Besançon (Centre de recherches d'histoire ancienne, 29).

2004. «La machine d'Héron et le coût de l'énergie dans le monde antique », in E. Lo Cascio (ed.), Innovazione tecnica e progresso economico nel mondo romano, Atti degli Incontri capresi di storia dell'economia antica (Capri 13-16 aprile 2003), à paraître dans la série Pragmateiai, Bari, Edipuglia. Breton, Philippe

1995. À l'image de l'homme. Du Golem aux créatures artificielles. Paris : Seuil (« Science ouverte »). 
Ceccarelli, Paola

1992. « Le monde sauvage et la cité dans la comédie ancienne », Études de Lettres $1:$ 23-37.

Chapuis, Alfred \& Edmond Droz

1949. Les Automates, figures artificielles d'hommes et d'animaux. Neufchâtel : Éditions du Griffon.

Chapuis, Alfred \& Edouard Gélis

1984. Le Monde des automates. Étude historique et technique. Genève : Éditions Slatkine (1ère édition

1928), 2 vol.

Chernyshov, Juri G.

1991-92. "From the "golden race" to the "golden age" : (some stages in the development of the ancient myth)», Archaiognosia 7 : 111-117.

Cohen, John

1968. Les Robots humains dans le mythe et dans la science. Paris : Vrin (« Études de psychologie et de philosophie, $19 »)$. [Ed. orig. Human Robots in Myth and Science, George Allen \& Unwin, Ltd, 1966].

Cook, Arthur Bernard

1914. Zeus, a Study in Ancient Religion. Cambridge : The University Press.

Delcourt, Marie

1982. Héphaïstos ou la légende du magicien. Paris : Les Belles Lettres (« Confluents

psychanalytiques »).

Deroy, Louis

1956. « À propos de l'épithète homérique d'Héphaïstos amphigyêeis », Revue de l'Histoire des

Religions $150: 129-135$.

Detienne, Marcel \& Jean-Pierre Vernant

1974. Les Ruses de l'intelligence. La mètis des Grecs. Paris : Flammarion.

Dumézil, Georges

1952. Les Dieux des Indo-européens. Paris : Presses universitaires de France.

Faraone, Christopher A.

1987. « Hephaestus the Magician and the Near Eastern Parallels for Alcinous' watchdogs ", Greek, Roman and Byzantine Studies 28 : 257-280 (repris et développé dans Talismans and Trojan horses: guardian statues in Ancient Greek myth and ritual, chap. 2. New York, Oxford : Oxford University Press, 1992).

Federico, Eduardo

1989. « Talos : funzione e rifunzionalizzazioni di un mito eteocretese », AION (archeol) 11 : 95-120.

Frontisi-Ducroux, Françoise

2000. Dédale. Mythologie de l'artisan en Grèce ancienne. Paris : La Découverte ( Sciences humaines et sociales») (1ère édition 1975).

2002. " "Avec son diaphragme visionnaire : iduiêisi prapidessi, Iliade XVIII, 481". À propos du bouclier d'Achille ", Revue des études grecques 115 : 463-484.

Germain, Gabriel

1954. Homère et la mystique des nombres. Paris : Presses Universitaires de France.

Gille, Bertrand

1983. Les Mécaniciens grecs. Paris : Seuil (« Science ouverte »). 
Griset, Emanuele

1965. «I cani del palazzo di Alcinoo nelle moderne traduzioni », Rivista di studi classici 13 :

180-182.

Humbach, Helmut

1969. « Amphiguos und amphiguêeis », pp. 569-578, in Studi linguistici in onore di Vittore Pisani, t.2. Brescia.

Judet de la Combe, Pierre

1996. «La dernière ruse : "Pandore" dans la Théogonie », pp. 263-300, in F. Blaise, P. Judet de la Combe et Ph. Rousseau (eds), Le Métier du mythe. Lectures d'Hésiode (Cahiers de Philologie, vol. 16, série Apparat critique). Lille : Presses universitaires du Septentrion.

Leclerc, Marie-Christine

1993. La Parole chez Hésiode. À la recherche de l'harmonie perdue. Paris : Les Belles Lettres

(« collection d'études anciennes »).

Lecourt, Dominique

2000. « Technophobie », Cités $4: 15-20$.

2003. Humain, posthumain. Paris : Presses Universitaires de France.

Leroy, Marie-Madeleine

1982. « À propos de Pieds d'or : la claudication du forgeron indo-européen en Europe

occidentale ", Ethnologie française $12: 291-296$.

Lloyd, Geoffrey E.R.

1993. Une histoire de la science grecque. Paris : Seuil («Textes à l'appui ») [Édition originale, Demystifiyng Mentalities, Cambridge, 1990].

Loraux, Nicole

1990. "Sur la race des femmes et quelques-unes de ses tribus », pp. 75-117, in Les Enfants d'Athéna. Idées athéniennes sur la citoyenneté et la division des sexes. Paris : Seuil.

"Le corps vulnérable d'Arès ", pp. 465-492, in Ch. Malamoud et

J.-P. Vernant (eds), Corps des dieux. Paris : Gallimard (1ère édition 1986).

Lostoriat Delabroise, Véronique

2000. « Le langage du corps dans l'Iliade », Kentron 17, 2 : 51-63.

Mainoldi, Carla

1984. L'Image du loup et du chien dans la Grèce ancienne d'Homère à Platon. Paris : Ophrys.

Martin, Dale B.

1995. The Corinthian Body. New Haven/London : Yale University Press.

Mehl, Véronique

2002. «Au plus près de l'autel, la circumambulation au cours des sacrifices ", Revue des Études

Anciennes 104, 1-2:25-49.

Morocho Gayo, Gaspar

1977. « La edad d'oro en Hesíodo y en la comedia antigua », Helmantica 28 :377-387.

Morris, Sarah P.

1992. Daidalos and the Origins of Greek Art. Princeton : Princeton University Press.

Muller-Dufeu, Marion

2002. La Sculpture grecque. Sources littéraires et épigraphiques. Paris : École nationale supérieure des beaux-arts (« Beaux-arts Histoire »). 
Musti, Domenico

2000. «L'automatismo in Omero : note sulla decorazione dello scudo d'Achille », pp. 401-409, in Homère chez Calvin. Figures de l'hellénisme à Genève. Mélanges Olivier Reverdin. Genève : Droz.

Onians, Richard Broxton

1999. Les Origines de la pensée européenne sur le corps, l'esprit, l'âme, le monde, le temps et le destin. Paris : Seuil (« L'ordre philosophique »). [Édition originale, The Origins of European thought about the Body, the Mind, the Soul, the World, Time and Fate. Cambridge University Press, 1951].

Padel, Ruth

1992. In and Out the Mind. Greek Images of the Tragic Self. Princeton (N. J.) : Princeton University

Press.

Peigney, Jocelyne

1991. «À propos de la vision grecque du corps humain », pp. 98-102, in Les Grecs, les Romains et nous. L'Antiquité est-elle moderne? Textes réunis et présentés par R.-P. Droit. Paris : Le Monde Éditions.

Pigeaud, Jackie

1995. L'Art et le vivant. Paris : Gallimard.

Poirier, Jacques (sous la direction de)

1996. L'Âge d'or. Dijon : Éditions Universitaires de Dijon.

Pralon, Didier

2003 « Tekhnê chez Homère ", Technologies/Idéologies/Pratiques, Revue d'anthropologie des connaissances 15-1 : 105-118.

Pucci, Pietro

1977 Hesiod and the Language of Poetry. The John Hopkins University Press.

Saintillan, Daniel

1996. « Du festin à l'échange : les grâces de Pandore », pp. 315-348, in F. Blaise, P. Judet de la Combe et Ph. Rousseau (eds), Le Métier du mythe. Lectures d'Hésiode (Cahiers de Philologie, vol. 16, série Apparat critique). Lille : Presses universitaires du Septentrion.

Snell, Bruno

1994. La Découverte de l'esprit. La genèse de la pensée européenne chez les Grecs. Paris : Éditions de l'Eclat (« Polemos ») [Édition originale, Die Entdeckung des Geistes. Studien zur Entstehung des europaïschen Denkens bei den Greichen, Vandenhoeck \& Ruprecht, Göttingen, 1975, 1946].

Verdenius, Willem Jacob

1987. Commentaries on Pindar, t. 1. Leiden : E.J. Brill (Mnemosyne, Suppl. 97).

Vernant, Jean-Pierre

1985. Mythe et pensée chez les Grecs. Paris : La Découverte.

1996a. «Les semblances de Pandora », pp. 381-392, in F. Blaise, P. Judet de la Combe et Ph.

Rousseau (eds), Le Métier du mythe. Lectures d'Hésiode (Cahiers de Philologie, vol. 16, série Apparat critique). Lille : Presses universitaires du Septentrion.

1996b. « Les semblances de Pandora », pp. 396-413, in Entre mythe et politique. Paris : Seuil.

Vidal-Naquet, Pierre

1975. «Le mythe platonicien du Politique, les ambiguïtés de l'âge d'or et de l'histoire », pp.

374-391, in J. Kristeva (ed.), Langue, discours, société. Pour Emile Benveniste. Paris : Seuil.

1983. Le Chasseur noir. Formes de pensées et formes de société dans le monde grec. Paris : La Découverte. 
West, Martin L.

1978. Hesiod Works and Days. Oxford : Oxford University Press.

Wathelet, Paul

2000. « Dieux et enchantements dans l'épopée homérique », pp. 169-184, in La Magie, tome $2:$ La Magie dans l'antiquité grecque tardive. Les mythes. Études rassemblées par A. Moreau et J.-Cl. Turpin. Université Montpellier III.

Yche-Fontanel, Françoise

2001. «Les boiteux, la boiterie et le pied dans la littérature grecque ancienne », Kentron 17-2 : 65-90.

\section{NOTES}

1. Définition donnée par le Petit Robert (1992).

2. Sur ces termes, voir Beaune (1980:7).

3. Rappelons-le, cette fable raconte l'histoire d'un homme qui imite un magicien et transforme un balai en serviteur, mais se révèle ensuite incapable d'arrêter le processus, multipliant la métamorphose.

4. Il s'agit du dialogue intitulé L'Amateur de mensonges.

5. Voir Chapuis \& Droz 1949, Chapuis \& Gélis 1984, Cohen 1968 : 9-21, et plus récemment Beaune (1980) Breton (1995 : 36-37 et 84-88); Amartin-Serin (1996 : 11-20).

6. Les principales sources littéraires sont l'Iliade 1, 571 sq.; 18, 369 sq.; Hésiode, Théogonie 927-929; Scholie à Homère, Iliade 14, 296; Apollodore, Bibliothèque 1, 3, 5. Pour une présentation générale d'Héphaïstos, voir Delcourt (1982); L. Malten, s.v. Hephaïstos, RE, VIII, 1, col. 311-366.

7. Khôlos : Iliade 18, 397; Odyssée 8, 308, 332; voir également Iliade 18, 411, 417; 20, 37; Héphaïstos est un dieu infirme, hêpedanos, Odyssée 8, 311. Sur l'infirmité d'Héphaïstos, on consultera d'une manière générale, Delcourt (1982 : 110 sq.); Detienne \& Vernant (1974 : chap. 9), puis plus particulièrement sur les pieds courbés du divin forgeron, Yche-Fontanel (2001); Leroy (1982); Deroy (1956). L'infirmité du dieu forgeron se retrouve en particulier dans la mythologie scandinave (Dumézil 1952).

8. Humbach (1969) comprend néanmoins ce terme comme « celui qui tient les tenailles de la forge ".

9. Sur les différentes variantes de la naissance d'Héphaïstos, voir Delcourt (1982 : chap. 2).

10. Hésiode, Théog. 929 : Héphaïstos est « le meilleur artisan de la race ouranienne » (trad. Ph. Brunet, LGF); une épithète souvent employée est klutotekhnês, « l'illustre artisan ", cf. Iliade 1, 571; 18, 143 et 391; Odyssée 8, 286; Hésiode, frag. 141,4. Sur la tekhnê dans les textes homériques, voir Pralon 2003.

11. Sur ce terme daidala, difficile à traduire, on renverra aux études de FrontisiDucroux (2000), Morris (1992).

12. L'Odyssée évoque également la fabrication d'un cratère $(4,615-617 ; 15,115-117)$ et d'une grande urne en or, propriété de Dionysos (24,74-75). Pour les autres ouvrages attribués à Héphaïstos, voir Delcourt (1982 : chap. 3).

13. Iliade 18, 373-377; voir également Aristote, Politique 1, 4, 1253b35-37; Philostrate, Vie d'Apollonios de Tyane 3, 27. 
14. Pausanias $10,5,12$, qui cite Pindare. La nature exacte de ces créations nous échappe cependant.

15. Odyssée 7, 91-94. On pourrait rapprocher ces créations d'un chien, en or ou en bronze selon les versions, qui aurait attiré la convoitise de Pandaréôs, et que le fils de Mérops aurait par la suite offert à Tantale : cf. Antoninus Liberalis, Métamorphoses 36; Scholie à Homère, Odyssée 19, 518 : «Pandaréôs déroba, dans le sanctuaire de Zeus en Crète, le chien animé (empsukhon) en or fabriqué par Héphaïstos et le confia en dépôt à Tantale "; Scholie à Homère, Odyssée 20, 66; Le scholiaste de Pindare, Olympique 1, 91a, glose sur la fonction prophylactique du canidé dans le temenos crétois de Zeus. $C f$. Faraone $1987: 260$.

16. Pollux, Onomasticon 5, 38; Apollodore, Bibliothèque 2, 4, 7.

17. Apollodore, Bibliothèque 1, 9, 23.

18. Nonnos, Dionysiaques 29, 197-201.

19. Hygin, Astronomiques 2, 15.

20. Apollodore, Bibliothèque 1, 9, 26; Apollonios de Rhodes, Argonautiques 4, 1638 sq. (et scholies); Simonide, frag. 568 Page ( $c f$. également Scholies de Platon, République 337a21sq. et 48-50); Agatharchide (De mari Erythraeo 7, 76) dans Photius, Bibliothèque 443b22-25; Eustathe, Commentaire de l'Odyssée 20, 302; Scholie à Homère, Odyssée 20, 320; Platon, Minos [Sp.], 320c5-8; Lucien, De saltatione 49; Souda, s.v. Sardanios gelôs; Zenobius, Centuria 5, 85, 20 sq.

21. Iliade 5, 749-751; 8, 393-395. Héphaïstos est celui qui fabrique des portes infranchissables pour les dieux, nous l'avons vu; nous pouvons donc lui attribuer également ces portes « automatiques ». Comme le remarque Eustathe, dans le commentaire des vers 749-751 du chant 5, le terme automatai a bien le sens de "seules, d'elles-mêmes, sans gardiens, comme si elles étaient capables de réflexion (aisthêtikai) ». 22. Voir notamment Odyssée 7, 94 : les chiens d'or et d'argent donnés à Alkinoos sont « immortels, à jamais exempts de vieillesse » (trad. Mugler). Sur l'immortalité des chiens d'Alkinoos, voir Eustathe, Commentaire de l'Odyssée 7, 94 et Griset (1965). Pour une discussion et une illustration de cet épisode mythologique sur une kylix à figures noires du VIe siècle, on consultera Mainoldi (1984 : 70-71).

23. Sur le mythe hésiodique des races, voir plus particulièrement Vernant (1985 : 17-106), ainsi que les analyses rassemblées dans un ouvrage collectif : Blaise et al. (1996). Sur l'âge d'or dans la pensée grecque, voir Poirier (1996), Chernyshov (1991-92), VidalNaquet (1975) repris dans Vidal-Naquet (1983: 361-380).

24. Hésiode, Trav. 112-113, trad. Philippe Brunet, LGF.

25. Les hommes d'aujourd'hui, la race de fer (vers 176 sq.), connaissent au contraire le labeur (ponos), la misère et les souffrances; obligés de travailler la terre pour se nourrir, ils doivent de plus subir les maladies qui d'elles-mêmes (automatoi) s'acharnent sans cesse sur les malheureux hommes (Trav. 103).

26. Les servantes d'or apparaissent comme des auxiliaires indispensables pour le corps infirme d'Héphaïstos (cf. Iliade 18, 417, à comparer avec 18, 411 et 20, 37 : elles se substituent aux « jambes grêles » du dieu afin de soutenir son corps et permettre son déplacement) et de ce fait partagent sa peine : elles « s'empressent, s'essoufflent (epoipnuon) pour étayer leur maître » $(18,412)$, de la même manière que celui-ci s'empressait (poipnuonta) pour servir les dieux rassemblés au banquet $(1,600)$. Sur la démarche et la motricité d'Héphaïstos, voir par ailleurs les remarques de Bolens (2000: chap. 2).

27. Voir également Philostrate, Vie d'Apollonios de Tyane 3, 27. 
28. Cf. Athénée, Deipnosophistes 6, 267 sq., en particulier $267 f$ et $268 \mathrm{a}$, dont nous reprenons des passages (trad. J. Wilgaux) qui citent les Thêria (les Bêtes sauvages) de Cratès, poète comique athénien du $5^{\mathrm{e}}$ siècle a.C.; $c f$. Cratès, Thêria, 16 , dans Poetce Comici Grceci, Kassel-Austin (1983, t. 4 : 92). Voir Morocho Gayo (1977) et Ceccarelli (1992). 29. Notons au passage qu'Héphaïstos ordonne, « contraint au travail » les soufflets (keleuein, $c f .18,469)$ et commande aux automates, de même que les maîtres donnent des ordres et commandent des travaux à leurs esclaves (cf. Iliade 6, 324, 491; Odyssée 1, 357...). Peut-être n'est-il pas inutile de remarquer également que le nombre vingt s'applique aux trépieds forgés par Héphaïstos comme à des groupes d'esclaves ou de captives mentionnées dans l'Iliade $(9,139$ et 281$)$ ou dans l'Odyssée $(20,158) ; c f$. Germain (1954 : 19).

30. Pollux, Onomasticon 5, 38 : ce chien est en effet aphuktos, « celui auquel nul ne peut échapper ». Cette particularité fut néanmoins la cause de sa perte, car lorsqu'il fut confronté au renard de Teumessos qui, quant à lui, ne pouvait être capturé, les dieux pétrifièrent les deux animaux pour résoudre cette contradiction. L'animé redevint donc inanimé. L'adjectif aphuktos est souvent employé en relation avec Héphaïstos, car nul ne peut se défaire de ses liens; cf. Odyssée 8, 299; Souda, s.v. Hêphaisteios desmos. Voir Detienne \& Vernant (1974 : 268).

31. En suivant la course du soleil. Sur ces circumambulations et leur fréquence, $c f$. Mehl (2002: 42-43).

32. Cf. Apollonios de Rhodes, Argonautiques, IV, 1654sq.

33. C'est ce que suggère le verbe rôomai qui s'applique plus aux servantes qu'au dieu Hephaïstos. Les créatures artificielles au service d'Héphaïstos préfigurent les robots intelligents allant d'Olimpia à C3PO, le célèbre robot de Star Wars. D'ailleurs, notons avec une pointe d'humour que le terme robot provient du tchèque robota signifiant « travail forcé ». Né sous la plume du dramaturge Karel Capek, il apparaît en 1920 dans la comédie utopiste Rossum's Universal Robots.

34. Marie Delcourt (1982 : 57) force l'interprétation des mots kopte de desmous en proposant d'y trouver des liens magiques; desmos est à prendre ici au sens de «clou » et non de « lien », cf. Scholie à Homère, Iliade 18, 379.

35. Voir également Iliade 1, 608 et 18, 380.

36. Onians (1999, voir le chap. 2 « Organes de la conscience » : 39-62 et particulièrement les pages $42-56)$.

37. Detienne \& Vernant (1974: 55) : l'image du cercle est tout à la fois associée à la démarche (et à la mètis) d'Héphaïstos et aux mécanismes des automates. Cf. également Frontisi-Ducroux (2000 : 139).

38. Voir notamment scholie à Homère, Iliade 18, 372.

39. Tous les détails de l'apparence d'Héphaïstos peuvent en effet être expliqués par les commentateurs à l'aide de l'analogie avec le feu; $c f$. Scholie à Homère, Iliade 18, 415. Voir également les remarques de Bolens (2000 : chap. 2).

40. Cf. également Eustathe, Commentaire de l'Odyssée 7, 91-94.

41. Eustathe, en bon connaisseur des auteurs anciens, renvoie dans cette allusion aux Rhodiens à un passage de la $7^{\mathrm{e}}$ Olympique de Pindare (v. 52). Cf. Bresson (1979 : 50-53, note 39, 64-67), ainsi que les commentaires de Verdenius (1987 : 71-74).

42. Dans la continuité des discussions antiques et byzantines évoquées par Eustathe, voir Frontisi-Ducroux (2002 : 472-73), contra Musti (2000), pour lequel, dans la description du bouclier d'Achille, les éléments du décor doivent être considérés comme 
véritablement animés, semblables en cela aux automates qui seuls nous occupent dans cet article.

43. Pollux, Onomasticon 5, 38, trad. J. Wilgaux. Citons ici les propos ironiques de Fontenelle, à propos de la théorie des animaux-machines de Descartes : « Mettez une machine de chien et une machine de chienne l'une près de l'autre et il en pourra résulter une troisième petite machine, au lieu que deux montres seront l'une auprès de l'autre toute leur vie, sans jamais faire de troisième montre. Or, nous trouvons par notre philosophie, Mme B. et moi, que toutes les choses qui, étant deux ont la vertu de se faire trois, sont d'une noblesse bien élevée au-dessus de la machine » (Euvres complètes, Paris, 1742, t. 1, p. 31, cité par Beaune, 1980 : 9). Fontenelle apprécierait donc à leur juste valeur les créations d'Héphaïstos.

44. Cf. Scholie à Homère, Iliade 18, 373 et 470; Scholie à Homère, Odyssée 20, 518; Simonide, frag. 568 Page. Voir également Plutarque, Vie de Marcellus 17, passage dans lequel l'évocation d'une sorte de monstre né de la science d'Archimède repose sur l'opposition du corps et de l'âme (psukhè/sôma).

45. Pour une présentation générale de l'évolution des théories grecques concernant le corps et l'âme, voir Martin (1995).

46. Federico (1989) offre une étude exhaustive des sources et des interprétations; voir également Frontisi-Ducroux (2000: 125-130).

47. Apollodore, Bibliothèque 1, 9, 26.

48. Voir cependant les doutes exprimés dans la postface de l'édition publiée en 2000, p. 220.

49. La plupart des commentateurs supposent que les vers d'Hésiode s'inspirent du passage de l'Iliade, à l'exception notable de West (1978:158), qui propose l'influence inverse.

50. Iliade 3,$63 ; 4,309 ; 9$, 513-514, 553; 10, 391; 13, 730; 16, 688; 20, 133; 24, 40-41, 358; Odyssée, 2, 92; 5, 23; 6, 320; 8, 78; 24, 474.

51. Remarquons cependant que, selon Jan Bremmer (1983: 57), aucune hypothèse étymologique proposée jusqu'à présent n'est pleinement satisfaisante.

52. Voir également Pucci (1977 : 88 sq.), Loraux (1990 : 86), Leclerc (1993 : 119-128).

53. Voir également Peigney (1991), Lostoriat Delabroise (2000).

54. Sur l'importance des termes noos, audê et sthenos dans la conception archaïque du vivant, voir Bremmer (1983); Morris (1992 : 215 sq.).

55. Les Grecs ont « transformé un jeteur de sorts en un artisan du métal »; voir également les remarques introductives de Wathelet (2000).

56. Ce point est mis en valeur par certaines scholies; $c f$. par exemple Odyssée 7,92 , où l'un des scholiastes glose ainsi l'expression homérique iduiêisi prapidessi, que nous avons déjà évoquée : tais epistêmonikôtatais boulais kai sunesesi : «par les réflexions et les connaissances les plus savantes ».

57. Les deux divinités peuvent intervenir de manière conjointe, en fonction de leurs propres compétences, comme nous le voyons dans le processus de création de Pandore : c'est bien alors à Héphaïstos qu'il incombe de pétrir la glaise et l'eau pour modeler l'être artificiel. L'intervention d'Athéna est postérieure. Voir également Odyssée 6, 232-235 (= 23, 159-162). Cf. Frontisi-Ducroux (2000: 62). Remarquons cependant une exception : dans un passage de Pindare déjà évoqué ( $7^{\mathrm{e}}$ Olympique, v. 52), seule Athéna est mentionnée en relation avec de possibles automates, que Verdenius (1987 : 71-74) interprète néanmoins comme des artefacts dédaliques. 
58. Sur la similitude entre le feu et l'être vivant, voir par exemple Platon, Phèdre 245e, Plutarque, Questions de table 7, 4, 703a-b et Questions romaines 75, 281f. Sur le souffle comme principe vital, voir Martin (1995 : 21 sq.).

59. Voir les réflexions stimulantes de Pigeaud (1995), notamment les chapitres 6 à 8 , ainsi que Bresson (1979 : 64 sq.).

60. Dédale n'apparaît qu'une fois dans l'Iliade, au chant 18 et à propos d'une place de danse à Cnossos. Selon Sarah Morris (1992), c'est seulement au Ve siècle que se forge la légende d'un Dédale sculpteur et architecte, qui trouve cependant son origine dans des influences proche-orientales. Sur l'animation des statues, voir les références rassemblées par Muller-Dufeu (2002 : n. 125 sq.).

61. Philostrate, Galerie de Tableaux 1, 16, 3.

62. Pline, Histoire naturelle 34, 75.

63. Aulu Gelle, Nuits attiques 10, 12, 9-10.

64. Pour une histoire de la science grecque, on se reportera à Gille (1983 : 83-145), Lloyd (1993 : 194-196 et 287-306). Sur Héron d'Alexandrie, on dispose maintenant du texte et de la traduction des Livres I et II par Argoud \& Guillaumin 1997. Voir également Bresson (2004).

\section{RÉSUMÉS}

Cette étude, consacrée aux automates fabriqués par Héphaïstos, interroge les rapports entre techniques et vivant, entre nature et artifice dans l'imaginaire grec archaïque. Fruits des «savantes réflexions" du divin forgeron, et non d'une action magique, ces automates et créatures artificielles servent les dieux et peuvent parfois être offerts aux mortels. Capables de se mouvoir et d'agir par eux-mêmes, ils sont à ce point perfectionnés qu'ils peuvent dans certains cas se reproduire. En cela, ils sont comparables à Pandora, autre création d'Héphaïstos, révélant ainsi que technique et vivant ne s'opposent pas, mais obéissent aux mêmes principes.

\section{Automata and artificial creatures of Hephaistus : between science and fiction.}

This study, devoted to the automata created by Hephaistus, questions the relationship between technique and living, between natural and artificial in the archaic Greek representations. Result of the " clever thoughts " of the divine blacksmith, and not of a magic action, these automata serve the gods and can sometimes be offered to the mortals. Able to move and act by themselves, they are so sophisticated that they can be able to reproduce. From this point of view, they are comparable with Pandora, another creation of Hephaistus, revealing thus that techniques and nature are not opposed, but obey the same principles.

Autómatas y criaturas artificiales de Hefaistos : entre ciencia y ficción.

Con el estudio de los autómatas fabricados por Hefaistos se cuestionan las relaciones entre las técnicas y lo viviente, entre naturaleza y artificio en el imaginario griego arcaico. Frutos de las «sabias reflexiones » del divino herrero y no de una acción mágica, aquellos autómatas sirven a los dioses y a veces se pueden ofrecer a los mortales. Son capaces de moverse y actuar por sí mismo y son tan perfectos que pueden reproducirse. Son entonces comparables con Pandora otra creación de Hefaistos- lo que significa que técnica y viviente no se oponen sino que obedecen a los mismos principios. 
INDEX

Mots-clés : Âge d'or, créatures artificielles, automates, forgeron, Héphaïstos, magie, mythes grecs

Keywords : artificial creatures, automata, blacksmith, gold age, Greek myths, Hephaistus, magic

\section{AUTEURS}

\section{ALEXANDRE MARCINKOWSKI}

Crescam, Maison de la recherche en sciences sociales, Université de Rennes 2, place du recteur Le Moal, 35043, Rennes Cedex

JÉRÔME WILGAUX

Département d'Histoire, Crescam, Université de Nantes 